\title{
Recent Advances in the Genetics of Systemic Sclerosis: Toward Biological and Clinical Significance
}

\author{
Benjamin D. Korman • Lindsey A. Criswell
}

Published online: 18 March 2015

(C) The Author(s) 2015. This article is published with open access at Springerlink.com

\begin{abstract}
Significant advances have been made in understanding the genetic basis of systemic sclerosis (SSc) in recent years. Genomewide association and other large-scale genetic studies have identified 30 largely immunity-related genes which are significantly associated with SSc. We review these studies, along with genomewide expression studies, proteomic studies, genetic mouse models, and insights from rare sclerodermatous diseases. Collectively, these studies have begun to identify pathways that are relevant to SSc pathogenesis. The findings presented in this review illustrate how both genetic and genomic aberrations play important roles in the development of SSc. However, despite these recent discoveries, there remain major gaps between current knowledge of SSc, a unified understanding of pathogenesis, and effective treatment. To this aim, we address the important issue of SSc heterogeneity and discuss how future research needs to address this in order to develop a clearer understanding of this devastating and complex disease.
\end{abstract}

Keywords Scleroderma - Systemic sclerosis - Autoimmune disease $\cdot$ Genetics $\cdot$ Single nucleotide polymorphisms . Genomics $\cdot$ Mouse models $\cdot$ Heterogeneity

This article is part of the Topical Collection on Scleroderma

B. D. Korman

Division of Rheumatology, Feinberg School of Medicine,

Northwestern University, Chicago, IL, USA

e-mail: benjamin-korman@northwestern.edu

L. A. Criswell $(\square)$

Rosalind Russell/Ephraim P. Engleman Rheumatology Research

Center, Division of Rheumatology, University of California, San

Francisco, CA, USA

e-mail: Lindsey.criswell@ucsf.edu

\section{Introduction}

Systemic sclerosis (SSc, the systemic form of scleroderma) is a complex disease with features that include autoimmunity, vasculopathy, and fibrosis. The disease is more common in women (4:1 female to male ratio) [1, 2]. There is significant clinical heterogeneity between SSc patients which remains poorly understood [3], and there remain critical gaps in understanding of the biologic basis of SSc. The core signs and symptoms of SSc are Raynaud's phenomenon, skin thickening, and serum autoantibody production, but patients have different patterns of internal organ involvement with variable presentations and outcomes $[4,5]$. SSc patients overall have an estimated $66 \%$ 10-year survival rate, which decreases to $38 \%$ for those with significant internal organ involvement $[6$, 7]. Pulmonary fibrosis and pulmonary artery hypertension (PAH) are leading causes of death and affect approximately $15 \%$ of SSc patients [8]. Cardiac disease including left- and right-sided heart failure, conduction system abnormalities, arrhythmias, or pericardial disease affects $15-35 \%$ of patients [9-12]. Skin involvement causes significant disability and also correlates directly with increased mortality [13]. The presence of cardiac involvement portends a poor prognosis with $70 \% 5$-year mortality [14] contributing to roughly $25 \%$ of SSc-related deaths [15].

SSc is a rare disease with an estimated 50 to 300 cases per million, and while the overall genetic burden is modest (only $2.6 \%$ of SSc patients' siblings develop SSc), evidence from familial, twin, and epidemiologic studies has implicated genetic predisposition for disease [16-19]. A positive family history raises relative risk by 15 - to 19 -fold in siblings relative to the general population, and first-degree relatives also have increased risk for developing Raynaud's phenomenon and interstitial lung disease [20, 21]. However, the genomic variants identified to date only account for approximately half of the genetic burden of SSc; environmental and epigenetic 
factors are thought to play a major role in this "missing heritability," and recent work has implicated multiple different epigenetic risk factors [22-24].

Like most autoimmune diseases, the genetic contribution to scleroderma is not due to a single rare genetic mutation but rather derives from many common genetic variants which predispose patients to disease. As an autoimmune disease, it has been well established that SSc is associated with HLA loci, and these studies have been recently reviewed elsewhere $[24,25]$. Through the use of high-throughput technologies including genomewide association studies (GWAS), researchers have identified and confirmed over 25 additional non-HLA SSc-associated genetic loci. The vast majority of these regions overlap with those that have been implicated in other autoimmune diseases. In this review, we will first briefly review this rapidly expanding area and then discuss other approaches that have implicated genetic pathways in $\mathrm{SSc}$ in an attempt to better understand disease pathogenesis.

\section{Immune Genes Implicated by Large-Scale Genetic Studies}

Prior to the past 10 years, beyond the HLA region, no clear SSc genetic susceptibility loci had been identified. However, with the advent of advances in genetic technologies and the development of national and multinational case-control cohorts, there have been an increasing number of studies that have identified significant genetic associations with systemic sclerosis. In this review, we will focus on loci that have been identified as genomewide significant and those that have been replicated.
Using genetic association results obtained for other autoimmune diseases including systemic lupus erythematosus and rheumatoid arthritis to identify candidate genes, significant associations have been identified between SSc and single nucleotide polymorphisms in the $B A N K 1, B L K, C D 226, I L 2 R A$, IL12RB, KCNA5, IRF5, STAT4, TNFAIP3, TNFSF4, and TLR2 genes [26-40].

The advent of GWAS allowed for confirmation of previously reported associations with the MHC region, IRF5, and STAT4 [41-43], and identified CD247 as a disease-associated locus [44]. Subsequent GWAS and GWA follow-up studies have identified IRF8 [43, 44], PSORS1C1 [45], IL12RB1 [46, 47], IL12RB2 [35], and CSK [39] loci as genomewide significant. In addition to these loci, at least two studies have confirmed significant association $\left(p<1 * 10^{-4}\right)$ at the TNFAIP3, TNFSF4, ATG5, SCHIP1-IL12A, and DNASE1L3 loci (Table 1). While the evidence confirming their association is not yet available, studies have now identified an additional 17 loci that have been demonstrated to have associations with $\operatorname{SSc}\left(p^{*} 10^{-4}>p>5 * 10^{-8}\right)$. These associations are summarized in Table 2, while Fig. 1 illustrates how many of these polymorphisms may contribute to disease pathogenesis.

While most of these studies have been extensively reviewed elsewhere [48, 49], three new studies in the past year have shed additional insights into the immunogenetics of SSc. In one of the largest genetic studies to date, Mayes et al. genotyped $1833 \mathrm{SSc}$ cases and 3466 controls with the Immunochip, a custom SNP genotyping array that provides high-density mapping of autoimmune disease-associated loci [50]. Using this approach, the authors identified novel

Table 1 Confirmed genomewide significant non-HLA associations or studies with two independent replications with $p<5^{*} 10^{-4}$

\begin{tabular}{|c|c|c|c|c|c|c|c|c|}
\hline Symbol & Gene name & Locus & SNP & Approach & Case/control & SSc phenotype & OR & $p$ value \\
\hline ATG5 [50] & Autophagy-related 5 & $6 q 25$ & rs9373839 & Immunochip & $1833 / 3466$ & $\mathrm{SSc}$ & 1.19 & $3.8 * 10^{-8}$ \\
\hline$C D 247[44,45]$ & $\mathrm{T}$ cell receptor zeta-chain & $1 \mathrm{q} 22$ & rs2056626 & GWAS & $2296 / 5171$ & $\mathrm{SSc}$ & 0.82 & $3.4 * 10^{-9}$ \\
\hline CSK [39] & c-src & $15 \mathrm{q} 24$ & rs 1378942 & GWA FU & $5270 / 8326$ & $\mathrm{SSc}$ & 1.20 & $5.0 * 10^{-12}$ \\
\hline DNASE1L3 [50] & Deoxyribonuclease I-like 3 & $3 \mathrm{p} 14$ & rs35646470 & Immunochip & $1833 / 3466$ & ACA (and all) & 2.03 & $4.3 * 10^{-31}$ \\
\hline IL12RB1 [47] & IL-12 receptor beta-1 & $19 \mathrm{p} 13$ & rs 2305743 & GWA FU & $8697 / 5032$ & $\mathrm{SSc}$ & 0.81 & $4.3 * 10^{-10}$ \\
\hline IL12RB2 [35] & IL-12 receptor beta-2 & $1 \mathrm{p} 31$ & rs3790567 & GWA FU & $3344 / 3848$ & $\mathrm{SSc}$ & 1.17 & $2.8 * 10^{-9}$ \\
\hline $\operatorname{IRF5}[44,45]$ & Interferon response factor 5 & $7 q 32$ & rs10488631 & GWAS & $2296 / 5171$ & $\mathrm{SSc}$ & 1.49 & $3.8^{*} 10^{-14}$ \\
\hline IRF8 [64] & Interferon response factor 8 & $7 \mathrm{p} 12$ & rs11642873 & GWAS & $3360 / 10,143$ & lcSSc & 0.75 & $2.3 * 10^{-12}$ \\
\hline PSORS1C1 [45] & Psoriasis susceptibility 1 candidate 1 & $6 \mathrm{p} 21$ & rs3130573 & GWAS & $564 / 1776$ & $\mathrm{SSc}$ & 1.25 & $5.7 * 10^{-10}$ \\
\hline SCHIP1-IL12A [50] & $\begin{array}{l}\text { Schwannomin interacting protein } \\
\text { 1/interleukin } 12 \text { alpha }\end{array}$ & $3 q 25$ & rs 77583790 & Immunochip & $1833 / 3466$ & $\mathrm{SSc}(\mathrm{lcSSc})$ & 2.57 & $1.2 * 10^{-11}$ \\
\hline STAT4 $[44,45]$ & $\begin{array}{l}\text { Signal Transducer and activator } \\
\text { of transcription } 4\end{array}$ & $2 \mathrm{q} 32$ & rs3821236 & GWAS & $2296 / 5171$ & $\mathrm{SSc}$ & 1.30 & $3.9 * 10^{-9}$ \\
\hline TNFAIP3 $[28,37]$ & TNF-associated interacting protein 3 & $6 \mathrm{q} 23$ & rs5029939 & CG & $1202 / 1196$ & $\mathrm{SSc}$ & 2.08 & $1.2 * 10^{-7}$ \\
\hline TNFSF4 [9, 31, 93] & TNF superfamily member 4 & $1 \mathrm{q} 25$ & rs 2205960 & CG & $1031 / 1014$ & $\mathrm{ACA}+$ & 1.33 & $1.3 * 10^{-5}$ \\
\hline
\end{tabular}

Genes that have been shown to be significant in two or more studies are in italics

lcSSC limited cutaneous systemic sclerosis, $S N P$ single nucleotide polymorphism, $O R$ odds ratio, $C G$ candidate gene, GWAS genomewide association study, GWA FU GWAS follow-up study, SSc systemic sclerosis, ATA anti-topoisomerase I antibody, $A C A$ anti-centromere antibody, SSCPAH SScassociated pulmonary arterial hypertension 
Table 2 Additional SSc genetic associations with one study with $p$ value between $5 * 10^{-4}$ and $5^{*} 10^{-8}$

\begin{tabular}{|c|c|c|c|c|c|c|c|c|}
\hline Symbol & Gene name & Locus & SNP & Approach & Case/control & $\begin{array}{l}\text { SSc } \\
\text { phenotype }\end{array}$ & OR & $p$ value \\
\hline BANK1 $[27,94]$ & B cell scaffold protein with ankyrin repeats 1 & $4 \mathrm{q} 24$ & rs 10516487 & CG & $1295 / 1137$ & $\mathrm{dcSSc}$ & 1.30 & $4.0 * 10^{-4}$ \\
\hline BLK/C8orf13 [32, 85] & $\begin{array}{l}\text { B lymphocyte kinase/chromosome } 8 \\
\text { open reading frame } 13\end{array}$ & $8 \mathrm{p} 23$ & rs 2736349 & CG & $1639 / 1416$ & $\mathrm{SSc}$ & 1.27 & $6.8 * 10^{-5}$ \\
\hline CD226 [33] & Cluster of differentiation 226 & $18 \mathrm{q} 22$ & rs763361 & CG & $1990 / 1642$ & $\mathrm{SSc}$ & 1.22 & $5.7 * 10^{-5}$ \\
\hline GRB10 [64] & Growth factor receptor-bound protein 10 & $7 \mathrm{p} 12$ & rs 12540874 & GWAS & $3360 / 10,143$ & lcSSc & 1.15 & $1.3 * 10^{-6}$ \\
\hline IL2RA [40] & IL-2 receptor alpha & $10 \mathrm{p} 15$ & rs2104286 & $\mathrm{CG}$ & $3023 / 2735$ & $\mathrm{ACA}+$ & 1.30 & $2.1 * 10^{-4}$ \\
\hline JAZF1 [51] & JAZF zinc finger 1 & $7 \mathrm{p} 15$ & rs1685352 & GWAS & $2761 / 3720$ & $\mathrm{SSc}$ & 1.14 & $3.6 * 10^{-5}$ \\
\hline KCNA5 [30] & $\begin{array}{l}\text { Potassium voltage-gated channel, } \\
\text { shaker-related subfamily, member } 5\end{array}$ & $12 \mathrm{p} 13$ & rs 10744676 & CG & $1576 / 1033$ & SScPAH & 0.64 & $3.0^{*} 10^{-4}$ \\
\hline KIAA0319L [51] & KIAA0319L & $1 \mathrm{p} 34$ & rs 2275247 & GWAS & $2761 / 3720$ & $\mathrm{SSc}(\mathrm{lc})$ & 1.46 & $3.9 * 10^{-6}$ \\
\hline NKFB1 [39] & Nuclear factor kappa beta 1 & $4 \mathrm{q} 24$ & rs1598859 & GWA FU & $5270 / 8326$ & $\mathrm{SSc}$ & 1.14 & $1.0 * 10^{-6}$ \\
\hline PPARG [52] & $\begin{array}{l}\text { Peroxisome proliferator-activated } \\
\text { receptor gamma }\end{array}$ & $3 \mathrm{p} 25$ & rs310746 & GWA FU & $2921 / 6963$ & $\mathrm{SSc}$ & 1.25 & $5.0^{*} 10^{-7}$ \\
\hline PSD3 [39] & Pleckstrin and Sec7 domain-containing 3 & $8 \mathrm{p} 22$ & rs10096702 & GWA FU & $5270 / 8326$ & $\mathrm{SSc}$ & 1.18 & $3.0 * 10^{-7}$ \\
\hline PXK [51] & PX domain-containing serine/threonine kinase & $3 \mathrm{p} 14$ & rs2176082 & GWAS & $2761 / 3720$ & $\mathrm{SSc}(\mathrm{ACA})$ & 1.21 & $4.4^{*} 10^{-7}$ \\
\hline RHOB1 [45] & Ras homolog family B & $2 \mathrm{p} 24$ & rs13021401 & GWAS & $564 / 1776$ & $\mathrm{SSc}$ & 1.21 & $3.7 * 10^{-6}$ \\
\hline RPL41 [64] & Ribosomal protein L41 & $12 q 13$ & rs11171747 & GWAS & $1699 / 10,143$ & $\mathrm{dcSSc}$ & 1.23 & $6.0 * 10^{-8}$ \\
\hline SOX5 [64] & Sex-determining region Y-box 5 & $12 \mathrm{p} 12$ & rs11047102 & GWAS & $1791 / 10,143$ & $\mathrm{ACA}+$ & 1.36 & $1.4^{*} 10^{-7}$ \\
\hline TLR2 [36] & Toll-like receptor 2 & $4 \mathrm{q} 32$ & rs5743704 & CG & $1622 / 1462$ & $\mathrm{SSc}$ & 2.24 & $3.0 * 10^{-4}$ \\
\hline TNIP1 [45] & TNFAIP3 interacting protein 1 & $5 q 32$ & rs2233287 & GWA FU & $4389 / 7611$ & $\mathrm{SSc}$ & 1.19 & $1.9 * 10^{-4}$ \\
\hline
\end{tabular}

associations at the DNASE1L3, SCHIP1-IL12A, and ATG5 loci [50]. Additionally, this work allowed dense HLA mapping stratified by antibody status (centromere and topoisomerase); using this large collection, and employing imputation and conditional analysis, they were able to identify a model composed of six polymorphic amino acid positions and seven SNPs which explains all observed associations in the HLA region in SSc and its serological subphenotypes. In a second study by Martin et al., the authors performed a meta-analysis of previous GWAS including both SSc and systemic lupus erythematosus (SLE) patients for a total of 6835 cases and 14,274 controls [51]. After replication of top hits in an independent SSc case-control study, this study identified novel SSc associations at KIAA0319L and the previously described SLE susceptibility loci $P X K$ and JAZF1. An additional GWA follow-up study identified a genomewide significant association of SSc with polymorphism near PPAR- $\gamma$ [52], a gene implicated in metabolism, immunity, and protection from fibrosis [53].

\section{Pathways and Potential Biomarkers Implicated by Human Transcriptomic and Proteomic Studies}

While genomic technologies have yielded major insights into genetic predisposition, utilization of other "omics" approaches, including transcriptomics and proteomics, has yielded additional important insights into pathways activated during SSc and in different patient subsets.

Microarray Intrinsic Subsets

Given the availability of skin as a target tissue in SSc, transcription profiling of skin biopsies from scleroderma patients has been undertaken and has yielded significant insights into scleroderma. Microarray studies have identified pathways that are activated in patients with disease and also identified novel patient subsets based on molecular expression patterns. Beyond the clinical classification of limited and diffuse SSc, the lack of biomarkers to explain patient heterogeneity has been a major limitation in identifying patients with different prognoses, and skin microarray has identified novel ways of evaluating SSc patients. Furthermore, the gene expression pattern has been shown to serve as a genetic biomarker for skin severity as a 44-gene subset can predict patients' modified Rodnan skin score (MRSS), a validated SSc-specific skin disease severity score [54, 55].

Skin biopsy microarrays are not only able to distinguish SSc from normal skin but also have proven helpful to classify SSc patients into fibroproliferative, inflammatory, limited, and normal-like subsets based on a core set of genes known as an "intrinsic subset" [54]. Michael Whitfield and colleagues have subsequently confirmed the validity of these subsets [55-58] and utilized them to better understand molecular stratification of patients. 


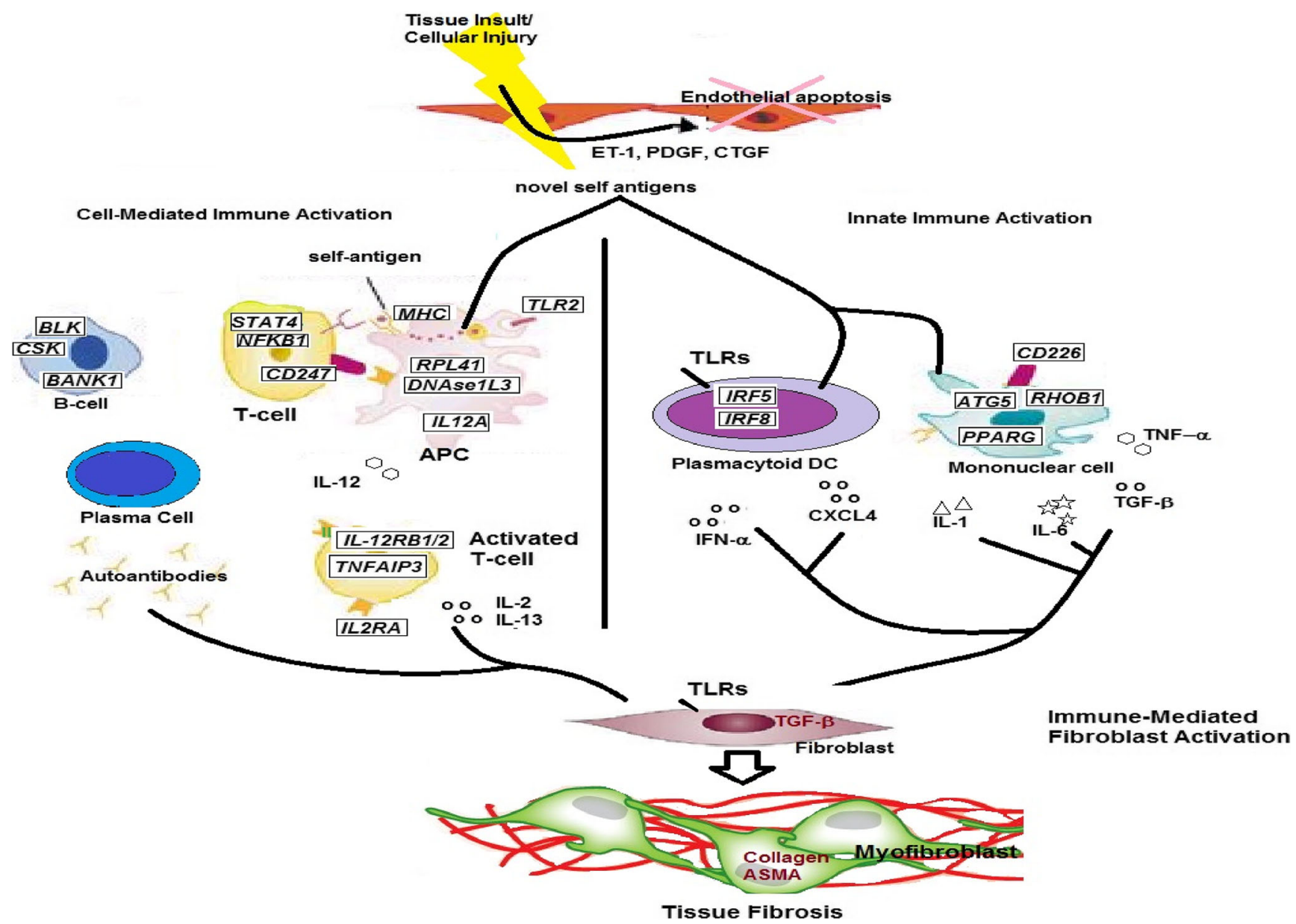

Fig. 1 Schematic of cellular roles for molecules genetically implicated in $\mathrm{SSc}$ pathogenesis. Tissue injury leads to release of self antigens and subsequent cell-mediated (via MHC) and innate (via TLRs) immune activation. Cells implicated in SSc and molecules genetically implicated in
SSc (italicized, boxed) are emphasized. Cell interaction and cell products lead to immune-mediated fibroblast activation and subsequent tissue fibrosis
The inflammatory group, marked by genes from the immune system response and inflammatory response Gene Ontology pathways, also includes upregulation of interferon-inducible genes, genes involved in vasculature development, and genes associated with fibrosis [54, 55]. This group tends to include patients with aggressive skin disease but more robust response to immunosuppressive treatment (mycophenolate mofetil) [58]. In contrast, the fibroproliferative group expresses genes from the mitosis, chromosome segregation, and DNA metabolic process pathways and tends to be more treatment refractory $[54,55,58]$. While initially thought to be intrinsic and identifying stable disease subsets [55], subsequent studies have shown that individual patients can change subsets both over time and in response to treatment with mycophenolate mofetil [58]. While not the primary pathway identified in unbiased analyses, the TGF- $\beta$ pathway has been shown to be activated in patients with a fibroproliferative intrinsic subset; this also correlates with downregulation of the PPAR- $\gamma$ pathway $[56,59]$.
Interferon-Inducible Signature

In peripheral blood and PBMCs, microarray studies have identified that roughly half of SSc patients possess an interferon signature similar to that seen in SLE and other autoimmune diseases [60-62]. Other studies have shown that plasmacytoid dendritic cells (pDCs) are the primary source of the interferon [63]. Given this finding, along with the association of SSc with polymorphisms in interferon regulatory factors $I R F 5$ and $I R F 8$ [44, 64], the interferon pathway may be playing a critical role in modulating SSc pathogenesis [65]. One study demonstrated that the plasma interferon score was higher in SSc patients than controls and correlated with Medsger disease severity index and pulmonary function parameters [66].

\section{CXCL4}

Proteomic analysis is still in its infancy but holds tremendous promise for the identification of potential biomarkers. In a 
recent study, proteomewide analysis showed that CXCL4 is the predominant protein secreted by pDCs in SSc, both in circulation and in skin [67]. The levels seen in SSc patients were substantially higher than those seen in other autoimmune diseases such as SLE and ankylosing spondylitis, higher in diffuse cutaneous than limited cutaneous disease, and higher in earlier dcSSc than in long-standing disease. Furthermore, levels correlated with skin and lung fibrosis and with pulmonary arterial hypertension, indicating that this may represent a novel disease-specific biomarker with prognostic significance.

In another study which used proteomics from pDCs to identify novel biomarkers, plasma levels of the Toll-like receptor agonist S100A8/9 were found to be elevated in SSc patients compared to controls [68].

\section{Insights From Rare Sclerodermatous Diseases}

\section{Cancer-Associated RNA Polymerase III Antibody SSc}

Anti-RNA polymerase 3 antibodies are observed in roughly $10 \%$ of SSc patients although prevalence is variable based on genetics and geography [69]. Joseph et al. performed an elegant study to determine whether RNA polymerase III antibodies may derive from cancer among the subset of SSc patients who develop them [70]. In previous studies, RNA pol III patients have been identified as being at a significantly increased risk of cancer and also of having a cancer diagnosis prior to or near the time of SSc diagnosis [71, 72]. Joseph et al. successfully isolated tumor DNA from histologic slides and identified mutations in the POLR3 gene or loss of heterozygosity in six of eight patients with cancer and RNA polymerase III antibodies and no patients with SSc and cancer with other autoantibodies [70]. Furthermore, immunologic characterization of the CD4+ T cells from patients with POLR3 tumor mutations demonstrated the presence of $\mathrm{T}$ cells reactive to the RPC1 peptide (an RNA polymerase III subunit encoded by POLR3) that are patient, peptide, and HLA specific.

\section{Stiff Skin Syndrome}

The stiff skin syndrome (SSS) is a rare Mendelian disorder caused by mutations in the fibrillin gene, the same gene responsible for Marfan syndrome, which is associated with highly elastic connective tissue. Unlike SSc, SSS does not portend other internal organ manifestations and is not associated with autoimmunity or vasculopathy [73]. Gerber et al. [74] attempted to recapitulate this disease utilizing a knockin strategy to create a strain of mice carrying a mutated fibrillin-1 allele identified in patients with SSS and another mutant mouse strain (D1545E) harboring an integrin mutation predicted to disrupt integrin binding to fibrillin-1. Both mutant mice strains developed dermal fibrosis accompanied by excess collagen deposition as well as progressive loss of intradermal adipose tissue. In addition to skin fibrosis, the transgenic mice spontaneously developed marked cutaneous inflammation, with accumulation of pDCs, Th2- and Th17skewed T helper cells, and plasma cells. Moreover, these mice developed circulating anti-topoisomerase I antibodies. Because fibrillin- 1 is known to modulate TGF- $\beta$ signaling and because the SSS fibrillin-1 mutations specifically affect the integrinbinding domain, the authors speculated that the stiff skin phenotype might be due to unchecked TGF- $\beta$ activation and increased TGF- $\beta$ signaling [74]. Interestingly, treatment of the mutant mice with a neutralizing antibody to TGF- $\beta$, as well as alpha-1 integrin-activating antibody, was able to reverse the fibrotic process and mitigate the immune dysregulation.

\section{Insights From Genetic Mouse Models of Scleroderma}

The most commonly utilized mouse model of scleroderma remains the bleomycin model which nicely recapitulates many of the seminal features of the disease, including fibrosis and inflammation. However, because this is a chemical injury model with largely unknown molecular mechanism(s), multiple attempts have been made to engineer or discover genetic mouse models that may yield important insights into disease pathogenesis. While none of the genetic models to date adequately recapitulates all disease features, the growing diversity of models enables researchers to study different aspects of disease and determine the effects of modulation of multiple relevant pathways on outcomes important in SSc. In Table 3, we summarize 11 different genetic models which appear to recapitulate important aspects of SSc.

\section{Tsk1 and Stiff Skin Syndrome Models: Fibrillin-1}

The tight skin mouse, which shows prominent spontaneous fibrosis of the hypodermis, has been widely used as a genetic mouse model of SSc, although exactly how this model corresponds with human disease has been difficult to explain. The Tsk1 mouse has now been shown to be caused by mutations in the fibrillin gene [75-77]. In the recent model by Gerber et al. discussed in "Stiff Skin Syndrome," knock-in human fibrillin1 mutations were introduced and the mice demonstrated a phenotype that included dermal fibrosis, inflammation, and autoimmunity. It is interesting that previous descriptions of human SSS have not described either a significant inflammatory component in the skin lesions nor described the autoimmunity that is seen in the mice. Whether this represents poor clinical characterization of human SSS due to its rarity or whether the mouse model more closely resembles SSc than SSS, the mouse model clearly recapitulates aspects of human fibrotic skin disease. It is not clear, however, whether it is a 


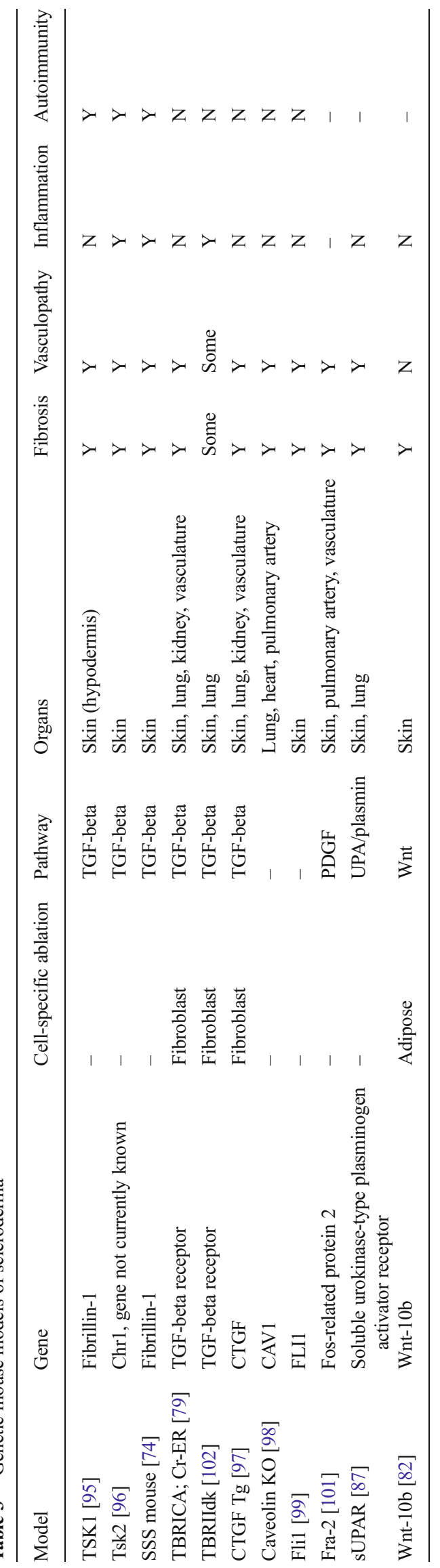

model of SSS, SSc, or a unique murine entity that lacks a human correlate.

Tsk2

The Tsk2 mouse may more closely mimic human disease (it spontaneously displays fibrosis, inflammation, and autoimmunity); while the ENU-induced locus is on chromosome 1, genetic studies are ongoing to elucidate the genetic lesion in this model [78].

\section{TGF- $\beta$ pathway}

TGF- $\beta$ is widely cited as the pathogenic molecule in SSc and other forms of fibrosis. Mice with an inducible constitutively active TGF- $\beta$ receptor I (TGF- $\beta$ RI) mutation driven by a fibroblast-specific promoter have been generated and demonstrate fibrosis of the dermis and fibrotic thickening of small blood vessel walls in the lung and kidney [79]. Primary skin fibroblasts from these mice showed elevated expression of downstream TGF- $\beta$ targets, reproducing the hallmark biochemical phenotype of explanted SSc dermal fibroblasts. Constitutive activation of TGF- $\beta$ in fibroblasts is therefore sufficient to induce a fibrotic phenotype.

Wnt and PPAR- $\gamma$ pathways

The Wnt pathway is a key developmental and homeostatic pathway in multiple tissues, and alterations in the pathway have been shown to be pro-fibrotic. Patients with SSc have increased fibroblast levels of $\beta$-catenin, a key Wnt mediator. Mice with fibroblast-specific $\beta$-catenin ablation rapidly develop skin fibrosis [80], while pharmacologic treatment with Wnt antagonists can reverse skin fibrosis [81]. Transgenic mice expressing Wnt-10b in adipose tissue showed not only progressive loss of subcutaneous adipose tissue but also dermal fibrosis, increased collagen deposition, fibroblast activation, and myofibroblast accumulation [82]. Wnt activity correlated with collagen gene expression in these biopsy specimens. This suggests that Wnt-10b switches differentiation of mesenchymal cells toward myofibroblasts by inducing a fibrogenic transcriptional program while suppressing adipogenesis.

Fibroblast-specific deletion of PPAR- $\gamma$, the master regulator of adipogenesis, results in enhanced susceptibility to bleomycin-induced skin fibrosis and enhanced sensitivity of fibroblasts to TGF- $\beta 1$ in PPAR- $\gamma$-deficient mice. These results indicate that PPAR- $\gamma$ suppresses fibrogenesis [83].

Fra-2 and sUPAR: Models of SSc Vasculopathy

The expression of the transcription factor Fra-2 is upregulated in SSc patients and in different mouse models of SSc. Fra-2 transgenic mice have been shown to develop spontaneous skin 
and lung fibrosis and vasculopathy (including skin microvasculopathy and pulmonary artery enlargement consistent with pulmonary hypertension) which are mediated by TGF- $\beta$ and PDGF [84]. This is particularly important because most other mouse models (both genetic and inducible) do not demonstrate significant vasculopathy which is a key clinical feature of SSc [86].

Urokinase-type plasminogen activator receptor (UPAR) has been implicated in SSc microvasculopathy. Mice deficient in soluble UPAR demonstrate skin fibrosis as well as decreased microvessels which were shown to have undergone apoptosis [87]. While these mice did demonstrate lung disease, it was more reminiscent of chronic pneumonia than of pulmonary fibrosis.

\section{SSc Heterogeneity and Genetics}

Nearly all of the genetic associations reviewed in "Immune Genes Implicated by Large-Scale Genetic Studies" represent genes involved in immunity, and associations with the same genes have been reported in multiple other autoimmune diseases. Despite the prominent fibrotic features of SSc, genetic studies to date have not identified major risk factors related to genes involved in the process of fibrosis [88]. Interestingly, recent studies have looked at SSc lung disease and idiopathic pulmonary fibrosis (IPF) and found little genetic overlap, suggesting that the fibrosis in SSc and ILD may be distinct [89, 90]. One explanation for the lack of fibrotic genes identified may be that the design of many of the studies, including the recent Immunochip and SSc-SLE pan-meta-GWAS, was biased toward identification of immune system loci. Because a core set of known fibrotic genes has not yet been identified, a similar strategy to enhance the identification of fibrotic genes is not as easily achievable. Furthermore, if fibrosis is secondary to either epigenetic changes or a process secondary to an aberrant immune response (see Fig. 1), that could explain the lack of fibrotic genes identified in otherwise largely agnostic GWAS.

Identification of genetic variants that contribute to SSc has been complicated by the complexity and heterogeneity of the disease. SSc behaves more like a syndrome than a unified disease as SSc patients can have multiple different clinical phenotypes and patterns of organ involvement. As personalized medicine advances, we may learn that there are in fact multiple or even numerous unique disease entities that are all currently classified as SSc. Cancer-associated RNA polymerase III antibody-associated SSc [70] is probably one example of molecularly defined disease that has a novel pathogenesis and may respond differently to treatment. For the purpose of understanding disease, heterogeneity likely hampers scientists' ability to identify genetic risk loci because cases represent patients with a multitude of SSc-related conditions.
The contribution of race/ethnicity represents another complicating factor; there are genetic differences between and across ethnicities which affect prevalence and relative importance of genetic susceptibility loci. Furthermore, Caucasian and Asian populations have been well represented in genetic studies compared to African-Americans, who have more severe SSc manifestations. Indeed, all of the large-scale genetic studies reviewed in "Immune Genes Implicated by LargeScale Genetic Studies" have focused on Caucasian populations, and further analysis of other ethnic groups may yield new genetic insights, as has been seen in other diseases [91].

Attempts to subclassify patients are frequently made on the basis of skin disease (limited versus diffuse cutaneous disease), autoantibodies, and organ involvement (particularly lung disease [3, 92]). There has already been substantial work to determine whether genetic associations are present only in certain SSc subtypes (mostly lcSSc/dcSSc and ATA/ACA). However, limited clinical phenotyping makes it difficult to study homogeneous SSc patient groups that represent disease endophenotypes and may have more distinct and clear-cut genetic predispositions.

More recently, the use of skin biopsy microarray intrinsic subsets as a way of classifying disease has been proposed as identifying disease endophenotypes [92]. Identification of additional novel biomarkers may further enable biological classification into subtypes, and that should hopefully contribute to better understanding of patients' genetic susceptibility to SSc. With functional studies such as gene expression analysis, another challenge for understanding SSc is studying specific classes of SSc cells and tissues. Because SSc has multiple diverse manifestations, it is difficult to determine which cell type is most appropriate for investigation. A great deal of focus has been on dermal fibroblasts, but whether these cells are primary or secondary in disease pathogenesis remains unclear. Similarly, while immune cells are clearly relevant, it remains unclear whether lymphocytes, dendritic cells, macrophages, or other cell types are most relevant. While the vasculature and lungs are clearly important, these tissues remain difficult to acquire. Skin biopsy remains an important tissue which is accessible and is of diagnostic/ prognostic significance, but because skin is very heterogeneous (keratinocytes, dermal fibroblasts, immune cells, adipocytes, vessels), analysis of gene expression may be obscured by genetic "noise" from multiple cell types. As cell-based assays and systems biology approaches develop further, it will be increasingly important to identify which cells to study and to study them in isolation in order to understand the biological relevance of SSc genetic associations. Figure 1 illustrates which cell types the identified genetic variants affect and how these may contribute to the pathogenesis of SSc.

With the advent of next-generation sequencing, there is also a movement toward personalized genomics. 
Despite the recent identification of a number of susceptibility loci in SSc as reviewed in "Immune Genes Implicated by Large-Scale Genetic Studies," other than HLA, the variants identified are not likely to be the causative variants. This is because GWAS utilize common (with the minor allele $>5 \%$ in the population) single nucleotide polymorphisms that are in linkage disequilibrium with implicated causative genetic variants. Furthermore, the total contribution of susceptibility loci discovered by GWA and follow-up studies explains only a fraction of the "heritability" of the disease. These concepts lead many to believe that there exist many rare variants with relatively large effects, which, in aggregate, account for the remaining prevalence of the disorder. These socalled private mutations can be identified with exome or whole genome sequencing and may be able to explain individual patients' genetic predisposition for disease. While such private mutations may be present in only one or very few affected individuals, identification of multiple private mutations within genes or in common pathways may also help elucidate disease pathogenesis that is more broadly relevant and inform the development of therapies that may help classes of patients.

While genetics clearly contributes importantly to SSc pathogenesis, it does not fully explain disease susceptibility. Environmental factors and associated epigenetic changes likely contribute importantly as well [23]. In addition to better epidemiologic studies to identify exposures such as chemicals and viruses that may influence disease risk, studies of epigenetic DNA modifications may also be crucial to understanding the molecular processes central to SSc.

SSc remains one of the most mysterious and difficult to treat diseases in modern medicine. While many studies have provided novel insights into genetic risk factors and pathways that are dysregulated, there remains a huge gap between current knowledge, understanding of pathogenesis, and the identification of effective treatments. The heterogeneity of SSc complicates its understanding, but modern omics technologies and better clinical phenotyping are contributing toward the goal of SSc being a disease that can be understood with systems biology tools and more effectively treated with a personalized medicine approach.

\section{Compliance with Ethics Guidelines}

Conflict of Interest Benjamin D. Korman and Lindsey A. Criswell declare that they have no conflicts of interest.

Human and Animal Rights and Informed Consent This article does not contain any studies with human or animal subjects performed by any of the authors.

Open Access This article is distributed under the terms of the Creative Commons Attribution License which permits any use, distribution, and reproduction in any medium, provided the original author(s) and the source are credited.

\section{References}

1. Krishnan E, Furst DE. Systemic sclerosis mortality in the United States: 1979-1998. Eur J Epidemiol. 2005;20(10):855-61.

2. Mayes MD, Lacey Jr JV, Beebe-Dimmer J, et al. Prevalence, incidence, survival, and disease characteristics of systemic sclerosis in a large US population. Arthritis Rheum. 2003;48(8):2246-55.

3. Steen VD. The pleomorphism of systemic sclerosis: are we ready for 'personalized medicine' in scleroderma? Expert Rev Clin Immunol. 2011;7(4):403-5.

4. Gabrielli A, Avvedimento EV, Krieg T. Scleroderma. N Engl J Med. 2009;360(19):1989-2003.

5. Varga J, Abraham D. Systemic sclerosis: a prototypic multisystem fibrotic disorder. J Clin Invest. 2007;117(3):557-67.

6. Steen VD, Medsger Jr TA. Severe organ involvement in systemic sclerosis with diffuse scleroderma. Arthritis Rheum. 2000;43(11): 2437-44.

7. Steen VD, Medsger TA. Changes in causes of death in systemic sclerosis, 1972-2002. Ann Rheum Dis. 2007;66(7):940-4.

8. Muangchan C, Canadian Scleroderma Research Group, Baron M, Pope J. The $15 \%$ rule in scleroderma: the frequency of severe organ complications in systemic sclerosis. A systematic review. J Rheumatol. 2013;40(9):1545-56.

9. Champion HC. The heart in scleroderma. Rheum Dis Clin North Am. 2008;34(1):181-90. viii.

10. Boueiz A, Mathai SC, Hummers LK, Hassoun PM. Cardiac complications of systemic sclerosis: recent progress in diagnosis. Curr Opin Rheumatol. 2010;22(6):696-703.

11. Kahan A, Coghlan G, McLaughlin V. Cardiac complications of systemic sclerosis. Rheumatology (Oxford). Jun 2009;48 Suppl 3: iii45-48.

12. Desai CS, Lee DC, Shah SJ. Systemic sclerosis and the heart: current diagnosis and management. Curr Opin Rheumatol. 2011;23(6): $545-54$.

13. Shand L, Lunt M, Nihtyanova S, et al. Relationship between change in skin score and disease outcome in diffuse cutaneous systemic sclerosis: application of a latent linear trajectory model. Arthritis Rheum. 2007;56(7):2422-31.

14. Medsger Jr TA, Masi AT. Epidemiology of systemic sclerosis (scleroderma). Ann Intern Med. 1971;74(5):714-21.

15. Tyndall AJ, Bannert B, Vonk M, et al. Causes and risk factors for death in systemic sclerosis: a study from the EULAR Scleroderma Trials and Research (EUSTAR) database. Ann Rheum Dis. 2010;69(10):1809-15.

16. Chifflot H, Fautrel B, Sordet C, Chatelus E, Sibilia J. Incidence and prevalence of systemic sclerosis: a systematic literature review. Semin Arthritis Rheum. 2008;37(4):223-35.

17. Englert H, Small-McMahon J, Chambers P, et al. Familial risk estimation in systemic sclerosis. Aust N Z J Med. 1999;29(1):36-41.

18. Jimenez SA, Derk CT. Following the molecular pathways toward an understanding of the pathogenesis of systemic sclerosis. Ann Intern Med. 2004;140(1):37-50.

19. Reveille JD. Ethnicity and race and systemic sclerosis: how it affects susceptibility, severity, antibody genetics, and clinical manifestations. Curr Rheumatol Rep. 2003;5(2):160-7.

20. Arnett FC, Cho M, Chatterjee S, Aguilar MB, Reveille JD, Mayes MD. Familial occurrence frequencies and relative risks for systemic sclerosis (scleroderma) in three United States cohorts. Arthritis Rheum. 2001;44(6):1359-62.

21. Frech T, Khanna D, Markewitz B, Mineau G, Pimentel R, Sawitzke A. Heritability of vasculopathy, autoimmune disease, and fibrosis in systemic sclerosis: a population-based study. Arthritis Rheum. 2010;62(7):2109-16. 
22. Broen JC, Radstake TR, Rossato M. The role of genetics and epigenetics in the pathogenesis of systemic sclerosis. Nat Rev Rheumatol. Aug 192014

23. Altorok N, Almeshal N, Wang Y, Kahaleh B. Epigenetics, the holy grail in the pathogenesis of systemic sclerosis. Rheumatology (Oxford). Apr 162014.

24. Luo Y, Wang Y, Wang Q, Xiao R, Lu Q. Systemic sclerosis: genetics and epigenetics. J Autoimmun. 2013;41:161-7.

25. Martin JE, Bossini-Castillo L, Martin J. Unraveling the genetic component of systemic sclerosis. Hum Genet. 2012;131(7):102337.

26. Dieude P, Guedj M, Wipff J, et al. The Ptpn22 620w allele confers susceptibility to systemic sclerosis: findings of a large case-control study of European Caucasians and a meta-analysis. Arthritis Rheum. 2008;58(7):2183-8.

27. Dieude P, Wipff J, Guedj M, et al. Bank1 is a genetic risk factor for diffuse cutaneous systemic sclerosis and has additive effects with Irf5 and Stat4. Arthritis Rheum. 2009;60(11):3447-54.

28. Dieude P, Guedj M, Wipff J, et al. Association of the TNFAIP3 rs5029939 variant with systemic sclerosis in the European Caucasian population. Ann Rheum Dis. 2010;69(11):1958-64.

29. Gourh P, Arnett FC, Tan FK, et al. Association of TNFSF4 (OX40L) polymorphisms with susceptibility to systemic sclerosis. Ann Rheum Dis. 2010;69(3):550-5.

30. Wipff J, Dieude P, Guedj M, et al. Association of a KCNA5 gene polymorphism with systemic sclerosis-associated pulmonary arterial hypertension in the European Caucasian population. Arthritis Rheum. 2010;62(10):3093-100.

31. Bossini-Castillo L, Broen JC, Simeon CP, et al. A replication study confirms the association of TNFSF4 (OX40L) polymorphisms with systemic sclerosis in a large European cohort. Ann Rheum Dis. 2011;70(4):638-41.

32. Coustet B, Dieude P, Guedj M, et al. C8orf13-BLK is a genetic risk locus for systemic sclerosis and has additive effects with BANK1: results from a large French cohort and meta-analysis. Arthritis Rheum. 2011;63(7):2091-6.

33. Dieude P, Guedj M, Truchetet ME, et al. Association of the CD226 Ser(307) variant with systemic sclerosis: evidence of a contribution of costimulation pathways in systemic sclerosis pathogenesis. Arthritis Rheum. 2011;63(4):1097-105.

34. Dieude P, Guedj M, Wipff J, et al. NLRP1 influences the systemic sclerosis phenotype: a new clue for the contribution of innate immunity in systemic sclerosis-related fibrosing alveolitis pathogenesis. Ann Rheum Dis. 2011;70(4):668-74.

35. Bossini-Castillo L, Martin JE, Broen J, et al. A GWAS follow-up study reveals the association of the IL12RB2 gene with systemic sclerosis in Caucasian populations. Hum Mol Genet. 2012;21(4): 926-33.

36. Broen JC, Bossini-Castillo L, van Bon L, et al. A rare polymorphism in the gene for Toll-like receptor 2 is associated with systemic sclerosis phenotype and increases the production of inflammatory mediators. Arthritis Rheum. 2012;64(1):264-71.

37. Koumakis E, Giraud M, Dieude P, et al. Candidate gene study in systemic sclerosis identifies a rare and functional variant of TNFA IP3 locus as a risk factor for individual polyautoimmunity. Arthritis Rheum. Apr 52012.

38. Manetti M, Allanore Y, Saad M, et al. Evidence for caveolin-1 as a new susceptibility gene regulating tissue fibrosis in systemic sclerosis. Ann Rheum Dis. 2012;71(6):1034-41.

39. Martin JE, Broen JC, Carmona FD, et al. Identification of CSK as a systemic sclerosis genetic risk factor through genome wide association study follow-up. Hum Mol Genet. 2012;21(12):2825-35.

40. Martin JE, Carmona FD, Broen JC, et al. The autoimmune diseaseassociated IL2RA locus is involved in the clinical manifestations of systemic sclerosis. Genes Immun. 2012;13(2):191-6.
41. Dieude P, Guedj M, Wipff J, et al. STAT4 is a genetic risk factor for systemic sclerosis having additive effects with IRF5 on disease susceptibility and related pulmonary fibrosis. Arthritis Rheum. 2009;60(8):2472-9.

42. Ito I, Kawaguchi Y, Kawasaki A, et al. Association of a functional polymorphism in the IRF5 region with systemic sclerosis in a Japanese population. Arthritis Rheum. 2009;60(6):1845-50.

43. Allanore Y, Dieude P, Boileau C. Updating the genetics of systemic sclerosis. Curr Opin Rheumatol. 2010;22(6):665-70.

44. Radstake TR, Gorlova O, Rueda B, et al. Genome-wide association study of systemic sclerosis identifies CD247 as a new susceptibility locus. Nat Genet. 2010;42(5):426-9.

45. Allanore Y, Saad M, Dieude P, et al. Genome-wide scan identifies TNIP1, PSORS1C1, and RHOB as novel risk loci for systemic sclerosis. PLoS Genet. 2011;7(7):e1002091.

46. López-Isac E B-CL, GGuerra S, Assassi S, Zhou X, PilarSimeón C, Ortego-Centeno N CI, Carreira P, Gorlova O, Beretta L, Santaniello A, Lunardi C HR, Nordin A, Riemekasten G, Witte T, Hunzelmann N, Kreuter A DJ, Voskuyl AE, deVries-Bouwstra J, Koeleman BP, Herrick A, Worthington J DC, Fonseca C, Radstake TR, Mayes MD, Martin J; the Spanish, Group. S. Identification of IL12RB1 as a novel systemic sclerosis susceptibility locus. Arthritis Rheumatol. 2014.

47. Lopez-Isac E, Bossini-Castillo L, S GG, et al. Identification of IL12RB1 as a novel systemic sclerosis susceptibility locus. Arthritis Rheumatol. Sep 82014.

48. Assassi S, Radstake TR, Mayes MD, Martin J. Genetics of scleroderma: implications for personalized medicine? BMC Med. 2013;11:9.

49. Mayes MD. The genetics of scleroderma: looking into the postgenomic era. Curr Opin Rheumatol. 2012;24(6):677-84.

50. Mayes MD, Bossini-Castillo L, Gorlova O, et al. Immunochip analysis identifies multiple susceptibility loci for systemic sclerosis. Am J Hum Genet. 2014;94(1):47-61.

51. Martin JE, Assassi S, Diaz-Gallo LM, et al. A systemic sclerosis and systemic lupus erythematosus pan-meta-GWAS reveals new shared susceptibility loci. Hum Mol Genet. 2013;22(19):4021-9.

52. Lopez-Isac E, Bossini-Castillo L, Simeon CP, et al. A genome-wide association study follow-up suggests a possible role for PPARG in systemic sclerosis susceptibility. Arthritis Res Ther. Jan 9 2014;16(1):R6.

53. Wei J, Bhattacharyya S, Jain M, Varga J. Regulation of matrix remodeling by peroxisome proliferator-activated receptor-gamma: a novel link between metabolism and fibrogenesis. Open Rheumatol J. 2012;6:103-15.

54. Milano A, Pendergrass SA, Sargent JL, et al. Molecular subsets in the gene expression signatures of scleroderma skin. PLoS One. 2008;3(7):e2696.

55. Pendergrass SA, Lemaire R, Francis IP, Mahoney JM, Lafyatis R, Whitfield ML. Intrinsic gene expression subsets of diffuse cutaneous systemic sclerosis are stable in serial skin biopsies. J Invest Dermatol. 2012;132(5):1363-73.

56. Sargent JL, Milano A, Bhattacharyya S, et al. A TGFbetaresponsive gene signature is associated with a subset of diffuse scleroderma with increased disease severity. J Invest Dermato. 2010;130(3):694-705.

57. Sargent JL, Whitfield ML. Capturing the heterogeneity in systemic sclerosis with genome-wide expression profiling. Expert Rev Clin Immunol. 2011;7(4):463-73.

58. Hinchcliff M, Huang CC, Wood TA, et al. Molecular signatures in skin associated with clinical improvement during mycophenolate treatment in systemic sclerosis. J Invest Dermatol. 2013;133(8): 1979-89.

59. Wei J, Ghosh AK, Sargent JL, et al. PPARgamma downregulation by TGFss in fibroblast and impaired expression and function in 
systemic sclerosis: a novel mechanism for progressive fibrogenesis. PLoS one. 2010;5(11):e13778.

60. Tan FK, Zhou X, Mayes MD, et al. Signatures of differentially regulated interferon gene expression and vasculotrophism in the peripheral blood cells of systemic sclerosis patients. Rheumatology (Oxford). 2006;45(6):694-702.

61. Higgs BW, Liu Z, White B, et al. Patients with systemic lupus erythematosus, myositis, rheumatoid arthritis and scleroderma share activation of a common type i interferon pathway. Ann Rheum Dis. 2011;70(11):2029-36.

62. Assassi S, Mayes MD, Arnett FC, et al. Systemic sclerosis and lupus: points in an interferon-mediated continuum. Arthritis Rheum. 2010;62(2):589-98.

63. Eloranta ML, Franck-Larsson K, Lovgren T, et al. Type i interferon system activation and association with disease manifestations in systemic sclerosis. Ann Rheum Dis. 2010;69(7):1396-402.

64. Gorlova O, Martin JE, Rueda B, et al. Identification of novel genetic markers associated with clinical phenotypes of systemic sclerosis through a genome-wide association strategy. PLoS Genet. 2011;7(7):e1002178

65. Wu M, Assassi S. The role of type 1 interferon in systemic sclerosis. Front Immunol. 2013;4:266

66. Liu X, Mayes MD, Tan FK, et al. Correlation of interferon-inducible chemokine plasma levels with disease severity in systemic sclerosis. Arthritis Rheum. 2013;65(1):226-35.

67. van Bon L, Affandi AJ, Broen J, et al. Proteome-wide analysis and CXCL4 as a biomarker in systemic sclerosis. N Engl J Med. 2014;370(5):433-43.

68. van Bon L, Cossu M, Loof A, et al. Proteomic analysis of plasma identifies the Toll-like receptor agonists $\mathrm{S} 100 \mathrm{a} 8 / \mathrm{A} 9$ as a novel possible marker for systemic sclerosis phenotype. Ann Rheum Dis. Apr 92014.

69. Sobanski V, Dauchet L, Lefevre G, et al. Prevalence of anti-RNA polymerase III antibodies in systemic sclerosis: new data from a French cohort and a systematic review and meta-analysis. Arthritis Rheumatol. 2014;66(2):407-17.

70. Joseph CG, Darrah E, Shah AA, et al. Association of the autoimmune disease scleroderma with an immunologic response to cancer. Science. 2014;343(6167):152-7.

71. Shah AA, Rosen A, Hummers L, Wigley F, Casciola-Rosen L. Close temporal relationship between onset of cancer and scleroderma in patients with RNA polymerase I/III antibodies. Arthritis Rheum. 2010;62(9):2787-95.

72. Moinzadeh $\mathrm{P}$, Fonseca $\mathrm{C}$, Hellmich $\mathrm{M}$, et al. Association of antiRNA polymerase III autoantibodies and cancer in scleroderma. Arthritis Res Ther. Feb 14 2014;16(1):R53.

73. Loeys BL, Gerber EE, Riegert-Johnson D, et al. Mutations in Fibrillin-1 Cause Congenital Scleroderma: Stiff Skin Syndrome. Sci Transl Med. Mar 17 2010;2(23):23ra20

74. Gerber EE, Gallo EM, Fontana SC, et al. Integrin-modulating therapy prevents fibrosis and autoimmunity in mouse models of scleroderma. Nature. 2013;503(7474):126-30.

75. Baxter RM, Crowell TP, McCrann ME, Frew EM, Gardner H. Analysis of the tight skin (Tsk1/+) mouse as a model for testing antifibrotic agents. Lab Invest. 2005;85(10):1199-209.

76. Saito S, Kasturi K, Bona C. Genetic and immunologic features associated with scleroderma-like syndrome of Tsk mice. Curr Rheumatol Rep. 1999;1(1):34-7.

77. Bona CA, Murai C, Casares S, et al. Structure of the mutant fibrillin1 gene in the tight skin (Tsk) mouse. DNA Res. 1997;4(4):267-71.

78. Long KB, Artlett CM, Blankenhorn EP. Tight skin 2 mice exhibit a novel time line of events leading to increased extracellular matrix deposition and dermal fibrosis. Matrix Biol. May 102014.

79. Sonnylal S, Denton CP, Zheng B, et al. Postnatal induction of transforming growth factor beta signaling in fibroblasts of mice recapitulates clinical, histologic, and biochemical features of scleroderma. Arthritis Rheum. 2007;56(1):334 44.

80. Beyer C, Schramm A, Akhmetshina A, et al. Beta-catenin is a central mediator of pro-fibrotic Wnt signaling in systemic sclerosis. Ann Rheum Dis. 2012;71(5):761-7.

81. Beyer C, Reichert H, Akan H, et al. Blockade of canonical Wnt signalling ameliorates experimental dermal fibrosis. Ann Rheum Dis. 2013;72(7):1255-8.

82. Wei J, Melichian D, Komura K, et al. Canonical Wnt signaling induces skin fibrosis and subcutaneous lipoatrophy: a novel mouse model for scleroderma? Arthritis Rheum. 2011;63(6):1707-17.

83. Kapoor M, McCann M, Liu S, et al. Loss of peroxisome proliferator-activated receptor gamma in mouse fibroblasts results in increased susceptibility to bleomycin-induced skin fibrosis. Arthritis Rheum. 2009;60(9):2822-9.

84. Reich N, Maurer B, Akhmetshina A, et al. The Transcription Factor Fra-2 Regulates the Production of Extracellular Matrix in Systemic Sclerosis. Arthritis Rheum. Jan 2010;62(1):280-90

85. Gourh P, Agarwal SK, Martin E, et al. Association of the C8orf13BLK region with systemic sclerosis in North-American and European populations. J Autoimmun Mar. 2010;34(2):155-62.

86. Maurer B, Distler JH, Distler O. The Fra-2 transgenic mouse model of systemic sclerosis. Vascul Pharmacol. 2013;58(3):194-201.

87. Manetti M, Rosa I, Milia AF, et al. Inactivation of urokinase-type plasminogen activator receptor (UPAR) gene induces dermal and pulmonary fibrosis and peripheral microvasculopathy in mice: a new model of experimental scleroderma? Ann Rheum Dis. Jul 12 2013.

88. Romano E, Manetti M, Guiducci S, Ceccarelli C, Allanore Y, Matucci-Cerinic M. The genetics of systemic sclerosis: an update. Clin Exp Rheumatol. 2011;29(2 Suppl 65):S75-86.

89. Borie R, Crestani B, Dieude P, et al. The MUC5B variant is associated with idiopathic pulmonary fibrosis but not with systemic sclerosis interstitial lung disease in the European Caucasian population. PLoS one. 2013;8(8):e70621.

90. Herzog EL, Mathur A, Tager AM, Feghali-Bostwick C, Schneider F, Varga J. Review: interstitial lung disease associated with systemic sclerosis and idiopathic pulmonary fibrosis: how similar and distinct? Arthritis Rheumatol. 2014;66(8):1967-78.

91. Fu J, Festen EA, Wijmenga C. Multi-ethnic studies in complex traits. Hum Mol Genet. 2011;20(R2):R206-213.

92. Varga J, Hinchcliff M. Connective tissue diseases: systemic sclerosis: beyond limited and diffuse subsets? Nat Rev Rheumatol. 2014;10(4):200-2.

93. Coustet B, Bouaziz M, Dieude P, et al. Independent replication and meta analysis of association studies establish TNFSF4 as a susceptibility gene preferentially associated with the subset of anticentromere-positive patients with systemic sclerosis. J Rheumatol May. 2012;39(5):997-1003.

94. Rueda B, Gourh P, Broen J, et al. Bank1 functional variants are associated with susceptibility to diffuse systemic sclerosis in Caucasians. Ann Rheum Dis. 2010;69(4):700-5.

95. Green MC, Sweet HO, Bunker LE. Tight-skin, a new mutation of the mouse causing excessive growth of connective tissue and skeleton. Am J Pathol. 1976;82(3):493-512.

96. Christner PJ, Ayitey S. Extracellular matrix containing mutated fibrillin-1 (Fbn1) down regulates Col1a1, Col1a2, Col3a1, Col5a1, and Col5a2 mRNA levels in Tsk/+ and Tsk/Tsk embryonic fibroblasts. Amino Acids. 2006;30(4):445-51.

97. Sonnylal S, Shi-Wen X, Leoni P, et al. Selective expression of connective tissue growth factor in fibroblasts in vivo promotes systemic tissue fibrosis. Arthritis Rheum. 2010;62(5):1523-32.

98. Drab M, Verkade P, Elger M, et al. Loss of caveolae, vascular dysfunction, and pulmonary defects in caveolin-1 gene-disrupted mice. Science. 2001;293(5539):2449-52. 
99. Asano Y, Stawski L, Hant F, et al. Endothelial Fli1 Deficiency Impairs Vascular Homeostasis: A Role in Scleroderma Vasculopathy. Am J Pathol. Apr 2010;176(4):1983-98.

100. Asano Y, Ihn H, Jinnin M, Tamaki K, Sato S. Altered dynamics of transforming growth factor beta(TGF-beta) receptors in scleroderma fibroblasts. Ann Rheum Dis. 2011;70(2): $384-7$.
101. Maurer B, Busch N, Jungel A, et al. Transcription factor fos-related antigen-2 induces progressive peripheral vasculopathy in mice closely resembling human systemic sclerosis. Circulation. 2009;120(23):2367-76.

102. Denton CP, Zheng B, Shiwen X, et al. Activation of a fibroblastspecific enhancer of the proalpha2(I) collagen gene in tight-skin mice. Arthritis Rheum. 2001;44(3):712-22. 